\title{
Calculation of beta-decay half-lives of proton-rich nuclei of intermediate mass
}

\author{
G. T. Biehle and P. Vogel \\ Physics Department, 130-33, California Institute of Technology, Pasadena, California 91125
}

(Received 26 June 1992)

\begin{abstract}
We present the results of a calculation of the beta-decay half-lives of several proton-rich even-even nuclei of intermediate mass: ${ }^{74} \mathrm{Sr},{ }^{76} \mathrm{Sr},{ }^{78} \mathrm{Zr},{ }^{80} \mathrm{Zr},{ }^{84} \mathrm{Mo},{ }^{86} \mathrm{Mo},{ }^{88} \mathrm{Ru},{ }^{90} \mathrm{Ru},{ }^{92} \mathrm{Pd}$, and ${ }^{96} \mathrm{Cd}$. The calculation is based upon the random phase approximation with the quasiparticle formalism and takes into account the residual particle-particle interaction.
\end{abstract}

PACS number(s): 23.40.Hc, 27.50. $+\mathrm{e}, 27.60 .+\mathrm{j}$

Ever since Takahashi et al. [1] calculated in 1973 estimates for the beta-decay half-lives of virtually all betaunstable nuclei, there has been a large effort to improve these estimates for neutron-rich nuclei because of applications in $r$-process theory and in the fate of fission products [2]. The theoretical effort to improve the estimates of half-lives of proton-rich nuclei has not been commensurate, although Hirsch et al. [3] and Muto et al. [4] presented calculations for light nuclei $(Z \leq 30)$. (See, however, also Refs. [5-7].) In this Brief Report we present the results of an effort to improve the estimates for half-lives of several proton-rich nuclei of intermediate mass. These half-lives play a role in $r p$-process theory [8], that is, the process in which protons are quickly added onto $\mathrm{C}, \mathrm{N}, \mathrm{O}$, and other "metals" with intervening fast positron decays resulting in heavy proton-rich nuclei. This process occurs in certain astrophysical contexts in which the temperature is greater than about $10^{8} \mathrm{~K}$. In particular, this process is predicted to occur in massive stars with degenerate neutron cores (if they exist) [9], and information about the longer-lived $(>1 \mathrm{~s})$ beta-unstable nuclei would allow one to predict the nuclear abundances on the surfaces of these stars [10]. For this reason we undertook the calculation of half-lives of some proton-rich even-even nuclei of intermediate mass.

We are interested in even-even nuclei which have $0^{+}$ ground states, so that the calculation is relatively simple. The positron-decay half-life $t_{1 / 2}$ is given by the following formula:

$$
\frac{1}{t_{1 / 2}}=\sum_{m} \frac{B(\mathrm{GT})_{m} g_{A}^{2}}{6160 \mathrm{~s}} f\left(\Delta E_{m}, Z\right),
$$

where $m$ labels the accessible $1^{+}$states in the daughter nucleus, $B(\mathrm{GT})_{m}$ is the Gamow-Teller $\beta^{+}$strength (equivalent to $\left|\left\langle m\left|\sigma \tau^{+}\right| i\right\rangle\right|^{2}$ in this case), $g_{A}$ is the axial-vector-current coupling constant (which we set to 1.25 ), and $f\left(\Delta E_{m}, Z\right)$ is the Fermi function (including Coulomb and relativistic corrections), which describes the size of phase space.

We obtain energy levels of the daughter nucleus and evaluate $B(G T)$ using the random phase approximation based on the quasiparticle formalism (QRPA). (The generalization of the QRPA to charge-changing modes is due to Halbleib and Sorensen [11]. Particle-particle interac- tions were first included in the QRPA by Cha [12].) The formalism is described in detail in Vogel and Zirnbauer [13] and in Engel et al. [14]. In these papers the authors use the $\delta$ force as the residual interaction and describe the following four parameters: $\alpha_{0}, \alpha_{1}$ (the particle-hole interaction constants in the $S=0$ and $S=1$ channels, respectively), $\alpha_{0}^{\prime}$, and $\alpha_{1}^{\prime}$ (the particle-particle interaction constants). Although these constants are theoretically related, the authors present an argument that they can be treated independently in this calculation. Using the values given in Ref. [14], we set $g_{\text {pair }}=-270 \mathrm{MeV} \mathrm{fm}^{3}$ when we solve the BCS equations, and we set $\alpha_{0}=-890$ $\mathrm{MeV} \mathrm{fm}{ }^{3}$ and $\alpha_{1}=-1010 \mathrm{MeV} \mathrm{fm}^{3}$ for the RPA portion of our calculations. Because we are looking at positron decay of proton-rich nuclei, our results do not depend on $\alpha_{0}^{\prime}$ in the RPA calculations. Our results do, however, depend strongly on the value of $\alpha_{1}^{\prime}$, so we must take care to choose it carefully.

We divide the nuclei into two categories, those with $74 \leq A \leq 80$ and those with $80<A \leq 96$. For the heavier nuclei in our study, we calibrated $\alpha_{1}^{\prime}$ using the known decay half-lives of ${ }^{88} \mathrm{Mo},{ }^{90} \mathrm{Mo},{ }^{92} \mathrm{Ru}$, and ${ }^{94} \mathrm{Pd}$. In order to calculate these half-lives, we identified the lowest-lying $1^{+}$state in the daughter nucleus with the ground state given by the QRPA calculation. (This determines the values of $\Delta E_{m}$, used in the phase space integrals.) Our calculation is for positron decay only, i.e., no electron capture. In three of the calibration nuclei positron decay dominates over electron capture; however, $75 \%$ of the decay of ${ }^{90} \mathrm{Mo}$ is due to electron capture. In that case we, therefore, use the proper partial decay rate. In our calculation, almost all ( $\gtrsim 90 \%)$ of the predicted decays occur into the lowest-lying $1^{+}$state. Figure 1 shows the $\log _{10}$ of the ratio of calculated positron-decay half-life to experimental half-life versus $\alpha_{1}^{\prime}$. From this figure we see that $\alpha_{1}^{\prime}$ may be anywhere within a window from -324 to $-333 \mathrm{MeV} \mathrm{fm}^{3}$ and yield values of half-lives correct to within a factor of 3 . A value of $\alpha_{1}^{\prime}=-329 \mathrm{MeV} \mathrm{fm}^{3}$ yields a least $\chi_{\text {red }}^{2}$ equal to 0.22 , where

$$
\chi_{\text {red }}^{2}=\left(\frac{1}{3} \sum\left[\log _{10}\left(T_{\text {calc }} / T_{\text {exp }}\right)\right]^{2}\right]^{1 / 2} .
$$

Thus we predict that our results in Table I are accurate to about a factor of $10^{0.22}=1.7$. By comparison, the $\chi_{\text {red }}^{2}$ 


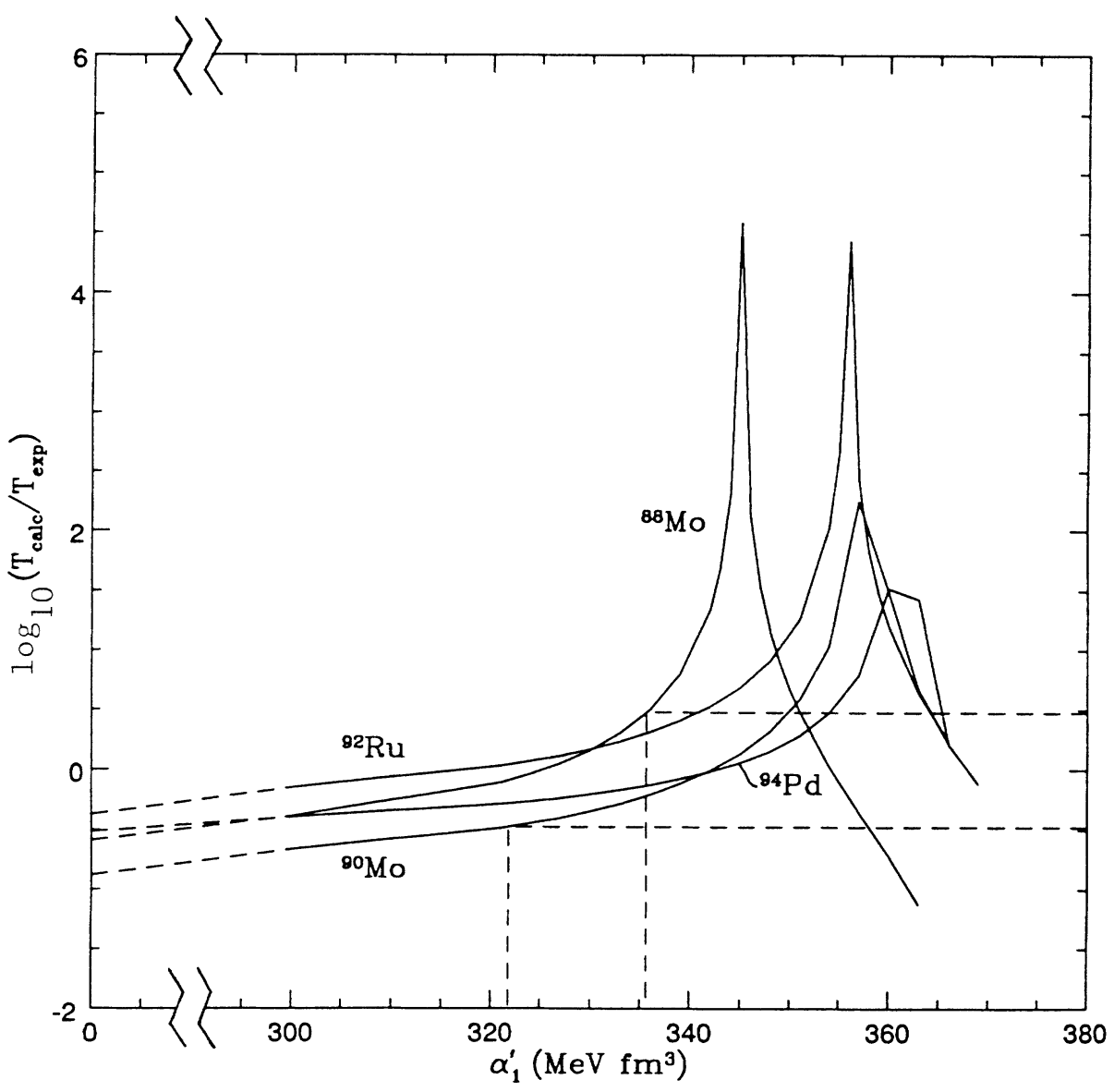

FIG. 1. $\log _{10}\left(T_{\text {calc }} / T_{\text {exp }}\right)$, where $T$ refers to the positron-decay half-life, versus $\alpha_{1}^{\prime}$ the particle-particle interaction strength. The window of values for $\alpha_{1}^{\prime}$ which yield results correct to within a factor of 3 is shown. Note that ignoring the residual particle-particle interaction (i.e., setting $\alpha_{1}^{\prime}$ to 0 ) results in prediction of half-lives approximately 3 to 10 times too small.

for these four nuclei using results from Takahashi et al. [1] is 0.59 , yielding an estimated accuracy of a factor of $10^{0.59}=4$.

In order to calculate half-lives of the nuclei listed in Table I, we need to know the positron-decay energies.

TABLE I. Predicted beta-decay half-lives.

\begin{tabular}{lccc}
\hline \multicolumn{4}{c}{ TABLE 1. Predicted beta-decay half-lives. } \\
$\begin{array}{lccc}\Delta E_{m=0} \text { a } \\
(\mathrm{MeV})\end{array}$ & $\begin{array}{c}\text { Half-life } \\
(\mathrm{s})\end{array}$ & $\begin{array}{c}\text { Takahashi et al. [1] } \\
\text { half-life (s) }\end{array}$ \\
\hline${ }^{74} \mathrm{Sr}$ & 9.6 & 0.5 & 0.03 \\
${ }^{76} \mathrm{Sr}$ & 4.5 & 8. & 3. \\
${ }^{78} \mathrm{Zr}$ & 10.5 & 0.06 & 0.03 \\
${ }^{80} \mathrm{Zr}$ & 5.0 & 7. & 3. \\
${ }^{84} \mathrm{Mo}$ & 5.2 & 6. & 0.8 \\
${ }^{86} \mathrm{Mo}$ & 3.9 & 90. & 16. \\
${ }^{88} \mathrm{Ru}$ & 5.8 & 1.2 & 0.8 \\
${ }^{90} \mathrm{Ru}$ & 4.7 & 16. & 5. \\
${ }^{92} \mathrm{Pd}$ & 6.8 & 0.9 & 0.4 \\
${ }^{96} \mathrm{Cd}$ & 8.0 & 0.6 & 0.3 \\
\hline \hline
\end{tabular}

${ }^{\text {a }}$ This is the maximum total energy of the positron for a transition to the lowest $1^{+}$daughter state.

${ }^{b}$ The estimated accuracy is a factor of 2 . See the explanation in the text.
Since the masses of the positron-decay parents (and often those of the daughters as well) are not known, we use the predicted masses of Jänecke and Masson [15]. (These seem to reproduce best the known masses of proton-rich nuclei.) We set $\Delta E_{m=0}$, that is, the maximum total energy of the positron, to the difference of parent and daughter masses less $0.2 \mathrm{MeV}$. The $0.2 \mathrm{MeV}$ represents a typical value for the energy difference between the ground state and the lowest-lying $1^{+}$state of the daughter nucleus. (For these decays, however, $\Delta E_{m=0}$ is large enough that the correction is trivial.) The results are shown in Table I. As stated in the previous paragraph, these values are accurate to within a factor of about 2. Electron capture is negligible in these nuclei, contributing less than $3 \%$ because of the large decay energies involved. (See Ref. [16].)

Similarly we use the known half-lives of ${ }^{70} \mathrm{Se},{ }^{72} \mathrm{Kr}$, ${ }^{74} \mathrm{Kr}$, and ${ }^{80} \mathrm{Sr}$ to calibrate $\alpha_{1}^{\prime}$ and calculate half-lives for several nuclei with $A \leq 80$. In this case we obtain $\alpha_{1}^{\prime}=-327 \mathrm{MeV} \mathrm{fm}^{3}$ for the best fit, yielding a least $\chi_{\text {red }}^{2}$ equal to 0.32 . The results are also shown in Table $I$. We estimate that the results are accurate to within a factor of about $10^{0.32}=2$, and again electron capture is negligible.

Also shown in Table I are the predicted half-lives of 
Takahashi et al. It is encouraging that our results are consistent with theirs, which are calculated by a different method; most of the difference is due to different $Q$ values (i.e., $\Delta E_{m=0}$ ), especially in the case of ${ }^{74} \mathrm{Sr}$.
The authors wish to acknowledge support from NASA Grant. No. NAGW-2920 and U.S. Department of Energy Contract No. DE-FG03-88ER40397.
[1] K. Takahashi, M. Yamada, and T. Kondoh, At. Data Nucl. Data Tables 12, 101 (1973).

[2] A. Staudt, E. Bender, K. Muto, and H. V. KlapdorKleingrothaus, At. Data Nucl. Data Tables 44, 79 (1990).

[3] M. Hirsch, A. Staudt, K. Muto, and H. V. KlapdorKleingrothaus, Nucl. Phys. A535, 62 (1991).

[4] K. Muto, E. Bender, and T. Oda, Phys. Rev. C 43, 1487 (1991).

[5] J. Suhonen, Phys. Lett. B 255, 159 (1991).

[6] I. N. Borzov, E. L. Trykov, and S. A. Fayans, Yad. Fiz. 52, 985 (1990) [Sov. J. Nucl. Phys. 52, 627 (1990)].

[7] A. Staudt, M. Hirsch, K. Muto, and H. V. KlapdorKleingrothaus, Phys. Rev. Lett. 65, 1543 (1990).

[8] R. K. Wallace and S. E. Woosley, Astrophys. J. Suppl. 45, 389 (1981).
[9] G. T. Biehle, Astrophys. J. 380, 167 (1991).

[10] G. T. Biehle (unpublished).

[11] J. A. Halbleib and R. A. Sorensen, Nucl. Phys. A98, 542 (1967).

[12] D. Cha, Phys. Rev. C 27, 2269 (1983); Ph.D. thesis, Michigan State Unversity, 1982.

[13] P. Vogel and M. R. Zirnbauer, Phys. Rev. Lett. 57, 3148 (1986).

[14] J. Engel, P. Vogel, and M. R. Zirnbauer, Phys. Rev. C 37, 731 (1988).

[15] J. Jänecke and P. J. Masson, At. Data Nucl. Data Tables 39, 265 (1988); see also pp. 289ff.

[16] C. M. Lederer and V. S. Shirley, Table of Isotopes, 7th ed. (Wiley, New York, 1978); see Appendix 21. 